\title{
La evaluación formativa como innovación. Experiencias en una Facultad de Formación del Profesorado
}

\author{
Marta Capllonch Bujosa ${ }^{1 *}$, Francesc Buscà Donet ${ }^{2}$ \\ Facultat de Formació del Professorat. Universitat de Barcelona
}

(Received September 30, 2011; Accepted January 15, 2012)

RESUMEN: La evaluación en las Facultades de Formación del Profesorado supone la creencia de que la forma es el contenido, y que los que nos movemos en este tipo de entornos formativos disponemos de mayores y mejores recursos para afrontar la evaluación en la universidad. Sin embargo, la evaluación como objeto de estudio requiere, en esta o en cualquier otra institución educativa la recopilación de evidencias conforme da respuesta a una necesidad, y se adecua a un determinado contexto.

En este trabajo se presenta el recorrido que ha realizado un grupo de innovación consolidado en evaluación formativa en la docencia universitaria de la Universidad de Barcelona, para llevar adelante sus sistemas de evaluación en diferentes grupos y asignaturas. Ciertamente, sus integrantes forman parte de estudios vinculados a la Formación de Maestros de Educación Primaria, pero no por ello se han formado o disponen de recursos que avalen una evaluación formativa, formadora, democrática y emancipadora como se requiere en el Espacio Europeo de Educación Superior.

En primer lugar se presentan unas breves pinceladas de lo que supone innovar en la docencia universitaria, y de la evaluación como objeto de innovación. A continuación se exponen las diferentes líneas de trabajo llevadas a cabo hasta el momento, y sobre todo, y lo más importante, a modo de conclusiones, se muestran las opiniones de los participantes en la experiencia, sobre el papel que juegan los grupos de innovación en el desarrollo profesional de los docentes.

Palabras clave: innovación, formación permanente, desarrollo profesional.

The formative assessment as innovation. Formative and shared assessment experiences in a Faculty of Teacher Training

\footnotetext{
ABSTRACT: For many people the assessment in Faculties of Teacher Training supposes the belief that form is content, and that those of us who move in this type of formative environments have more and better resources to deal with the assessment in faculty. However the assessment as object of study requires in this or in any other educational institution the gathering of evidences as a response to a need and as adaptation in a given context.

In this paper we present the trajectory that has made a consolidated innovation group in formative assessment in university teaching at the University of Barcelona, in order to further their assessment systems in different groups and sub- 
jects. It is true that its members are part of studies related to Teacher Training in Primary Education, although it does not mean that they have been trained or they have the resources to endorse a formative, democratic and emancipatory assessment as required in the European Higher Education Area.

First we present a brief summery of what is innovation in university teaching, and assessment as an object of innovation. Then we explain the different lines of work carried out so far. Above all and most importantly we expose, by means of conclusion, what the group members consider that happens to those who live with innovation throughout their teaching.

Keywords: innovation, lifelong learning, professional development.

\section{INTRODUCCIÓN: UNAS PALABRAS SOBRE INNOVACIÓN EN EL ÁM- BITO UNIVERSITARIO}

El concepto innovación implica diseñar y aplicar acciones orientadas a la trasformación y mejora de los procesos de enseñanza y aprendizaje. En el caso que nos ocupa se trata de un concepto que se suele utilizar para referirse a propuestas de corte didáctico proyectadas con la intención de resolver los problemas prácticos que surgen en el aula. Tal y como destaca De la Torre (1997), en función del enfoque paradigmático de referencia (técnico, cultural o social), estas propuestas son susceptibles de ser diseñadas, administradas, aplicadas y evaluadas por agentes internos o externos al contexto en el cual se lleva a cabo la innovación.

En la década de los 80 se consideraba que la innovación y el cambio se producían de forma personal a través de las modificaciones realizadas por determinados profesores. Algunos autores como Gros (2006), señalan incluso que los términos "innovación" y "cambio" eran utilizados con frecuencia de manera indiferente sin reparar en lo que encerraba la expresión. Progresivamente la creciente institucionalización de los esfuerzos por innovar dio paso a lo que en los últimos años Hannan y Sylver (2005, pp. 161-162) denominaron "innovación guiada", según la cual no se niega el proceso personal, pero se centra la atención en la innovación llevada a cabo en el aula, dirigida e incentivada por las instituciones. En efecto como señalan Gros y Lara (2009) la innovación no puede ser una práctica aislada. Un proyecto de innovación responde a una necesidad de diferenciación estratégica. La diferenciación es la meta y la innovación es el proceso para conseguirla. Por consiguiente, para que una organización sea innovadora ha de sistematizar y ejecutar de forma consciente y controlada su desarrollo estratégico. Los términos riesgo, confianza, colaboración, diferenciación, sostenibilidad, valor, calidad, son parte indisociable de la innovación.

En definitiva la innovación en la enseñanza y el aprendizaje, -y por ende en la evaluación como parte del proceso- es un estudio de interacciones, actitudes, normativas y prácticas institucionales, contextos nacionales y características de todos ellos. "Estimular la innovación" significa preguntar no sólo por el tipo de 
innovación, sino también: ¿Para quién, en interés de quien se innova y qué normativa está detrás? (Hannan y Silver, 2005, pp. 162).

Un proceso innovador, como nos recuerda Gros (2006) implica intención, planificación y esfuerzo e incluso así puede fracasar en los resultados. Por este motivo la investigación es especialmente relevante en tanto que permite dirigir los procesos de innovación a través del conocimiento generado por la investigación. Además, también forma parte de la investigación analizar los resultados de la innovación para valorar los resultados obtenidos.

\section{LA EVALUACIÓN COMO OBJETO DE INNOVACIÓN EN LA FORMA- CIÓN DEL PROFESORADO}

La creación del Espacio Europeo de Educación Superior (EEES) se ha convertido en una oportunidad no sólo para debatir, cuestionar y reflexionar, sino también para avanzar en la concreción de prácticas educativas y en el intercambio de experiencias que favorezcan una enseñanza y aprendizaje que mejore la educación superior a nivel supranacional (Margalef y Álvarez Méndez, 2005). La convergencia europea ha generado un necesario debate sobre el sistema formativo utilizado por las universidades. Se hacía necesaria y urgente la búsqueda de nuevas estrategias metodológicas más acordes con la formación que debe recibir un estudiante universitario.

Con objeto de dar respuesta a estas nuevas necesidades en los últimos años, la mayor parte de las universidades ha creado vicerrectorados relacionados con la innovación en la docencia y ha generado servicios que han adoptado nombres tan diversos como los de centro de innovación, servicio de innovación, gabinete de innovación... A modo de ejemplo la universidad de Barcelona dispone del Programa de Mejora e Innovación Docente (PMID) que contribuye a que uno de los objetivos prioritarios de la Universidad sea la docencia de calidad, generando la posibilidad de creación de grupos de profesores promovidos por intereses específicos. En concreto sus objetivos son:

- Estimular la innovación docente mediante ayudas económicas y apoyo metodológico a proyectos de innovación docente.

- Estructurar y cohesionar al profesorado implicado en la mejora de la docencia mediante el reconocimiento y la consolidación de grupos de innovación docente.

- Contribuir a la difusión, intercambio y extensión de las experiencias de mejora e innovación docente, a través de la página web del programa, la organización de actividades de intercambio de experiencias, y la presentación de los diferentes grupos y sus proyectos en el Observatorio de la Innovación Docente (OID).

En la mayoría de los casos, la innovación se asocia a cambios metodológicos muy relacionados con el EEES, y con el soporte en el uso de las TIC en la 
formación (Gros y Lara, 2009). No obstante, en los últimos años, la evaluación se ha convertido en uno de los principales tópicos en torno a los cuales se concretan los programas de innovación impulsados desde las universidades y las administraciones estatales y autonómicas, como así lo demuestran las convocatorias de innovación docente convocadas en el año 2011 por diez universidades públicas escogidas al azar donde se pone de manifiesto que la evaluación es un tema de actualidad en la promoción de proyectos de innovación docente, y que, tal y como se refleja en la tabla 1, los tópicos más recurrentes en este campo se refieren a: la mejora de los sistemas de evaluación en el marco de las nuevas titulaciones de grado, la evaluación de competencias, el diseño y la aplicación de instrumentos de evaluación orientados a la mejora del aprendizaje y el rendimiento académico en la universidad.

Tabla 1. Principales Tópicos Sobre Evaluación en los Proyectos en Docencia Universitaria

\begin{tabular}{|c|c|c|}
\hline $\begin{array}{l}\text { Comunidad } \\
\text { Autónoma }\end{array}$ & Universidad & $\begin{array}{l}\text { Tópicos relacionados con la } \\
\text { evaluación }\end{array}$ \\
\hline Andalucía & UCA & $\begin{array}{l}\text { Evaluación en el marco de la } \\
\text { aplicación de las nuevas titulaciones } \\
\text { de grado. }\end{array}$ \\
\hline Cantabria & UCAN & $\begin{array}{l}\text { - Mejora de los sistemas de evaluación } \\
\text { en las titulaciones de grado. }\end{array}$ \\
\hline Castilla La Mancha & UCLM & $\begin{array}{l}\text { - Evaluación de competencias } \\
\text { genéricas y específicas. }\end{array}$ \\
\hline Castilla-León & Valladolid & $\begin{array}{l}\text { - Sistemas de evaluación centrados } \\
\text { tanto en el proceso como en el } \\
\text { resultado. } \\
\text { - Diseño de instrumentos de evaluación } \\
\text { de carácter formativo. } \\
\text { - Desarrollo y evaluación de } \\
\text { competencias. }\end{array}$ \\
\hline Catalunya & UB & $\begin{array}{l}\text { - Desarrollo y adquisición de } \\
\text { competencias transversales. } \\
\text { - Diseño y aplicación de sistemas } \\
\text { de evaluación orientados al } \\
\text { aprendizaje y a la mejora del } \\
\text { rendimiento académico. }\end{array}$ \\
\hline Madrid & UAB & $\begin{array}{l}\text { - Sistemas de mejora del rendimiento } \\
\text { académico. }\end{array}$ \\
\hline Murcia & UCM & $\begin{array}{l}\text { - Estudio y diseño de procesos de } \\
\text { evaluación relacionados con la } \\
\text { innovación metodológica. } \\
\text { - Innovación en estrategias de } \\
\text { evaluación. }\end{array}$ \\
\hline \multirow{3}{*}{ Valencia } & UAM & $\begin{array}{l}\text { - Impulso del aprendizaje en red a } \\
\text { través de las TIC. }\end{array}$ \\
\hline & Murcia & $\begin{array}{l}\text { - Nuevas metodologías docentes y } \\
\text { de evaluación. } \\
\text { - Definir criterios y diseñar pruebas } \\
\text { de evaluación. }\end{array}$ \\
\hline & UV & $\begin{array}{l}\text { - Diseño de metodologías de } \\
\text { evaluación. }\end{array}$ \\
\hline
\end{tabular}


Por otra parte las bases de las convocatorias consultadas también aluden al desarrollo de los planes de estudio articulados sobre la base de las competencias profesionales, y a la implantación progresiva de un enfoque de los procesos de enseñanza y aprendizaje centrados en las y los estudiantes. Ambos casos ponen de manifiesto la poca pertinencia del uso exclusivo de las prácticas tradicionales de evaluación, puesto que ellas solas no son capaces de atender las finalidades y principios pedagógicos que el nuevo sistema universitario trae consigo. Todo ello justifica la necesidad de renovar el enfoque de la evaluación en la universidad y, por extensión, el diseño y la aplicación en las aulas universitarias de nuevas prácticas de evaluación.

\section{LÍNEAS Y PRINCIPALES RESULTADOS DE LOS PROYECTOS DE EVA- LUACIÓN FORMATIVA APLICADOS EN LA FORMACIÓN DEL PROFESORADO: EL CASO DE LA UB}

En el ámbito de la formación del profesorado, la necesidad de formar profesionales capaces de desarrollar el currículum de la enseñanza obligatoria y, por ende, los principios y finalidades de la sociedad del conocimiento en la que se amparan, guarda estrecha relación con la aplicación de sistemas de evaluación formativos, formadores y participativos. Con esta finalidad la Facultad de Formación del Profesorado de la Universidad de Barcelona está promoviendo desde el curso académico 2006-2007 la puesta en práctica de diversos proyectos de innovación docente, cuya principal intención es aplicar y valorar los efectos de los sistemas alternativos de evaluación implantados en el marco de varias asignaturas de las nuevas titulaciones de grado que se derivan del EEES.

Se trata de proyectos de evaluación formativa y participativa generados en torno a los siguientes objetivos:

- Describir los sistemas de evaluación aplicados en diferentes situaciones de enseñanza y aprendizaje en la formación del profesorado.

- Contrastar las experiencias de evaluación formativa aplicadas en diferentes titulaciones y asignaturas valorando su posible transferencia a otros contextos universitarios.

- Recopilar las prácticas de evaluación llevadas a cabo con éxito y que han superado los problemas y obstáculos detectados antes y durante su aplicación.

Estos proyectos se han gestado en el seno de un grupo de innovación consolidado por la Universidad de Barcelona. Para la obtención de este reconocimiento, los integrantes del grupo además de coordinar o participar en el desarrollo de estos proyectos, se han dedicado a diseminar sus resultados en publicaciones y congresos especializados. El requisito indispensable para participar en estos proyectos ha sido llevar a cabo un proyecto o caso de innovación en el aula universitaria centrado en la aplicación de sistemas de evaluación formativa. Si bien 
en un principio el profesorado participante en estos proyectos se adscribía a un único departamento del centro, a lo largo de estos cinco años de funcionamiento del grupo de innovación - desde su constitución y reconocimiento hasta su consolidación- se ha ido incorporando profesorado de diversos departamentos. La principal consecuencia de ello ha sido la diversificación de áreas de conocimiento implicadas y por extensión de sistemas de evaluación formativa.

El análisis de contenido de los informes valorativos elaborados por los miembros del los grupos de innovación, una vez concluidos sus proyectos, ponen de manifiesto gran parte de las ventajas e inconvenientes que recogen las experiencias de los miembros de la Red Interuniversitaria de Evaluación Formativa (López Pastor; Martínez-Muñoz y Julian, 2007; Vallés, Ureña, y Ruiz, 2011; Zaragoza, Luis, y Manrique, 2008). A saber:

- El profesorado considera que los sistemas de evaluación formativa y participativa permiten conocer mejor al alumnado y, por extensión, la obtención de informaciones mucho más relevantes sobre su proceso aprendizaje. Todo ello les permite, a su vez, emitir valoraciones y calificaciones mucho más pertinentes y ajustadas a la realidad.

- El docente obtiene informaciones acerca de las fortalezas y debilidades de sus estudiantes. Este hecho es fundamental para reorientar mejor su proceso de aprendizaje.

- El seguimiento más cercano y continuado del proceso de aprendizaje del alumnado posibilita que el profesorado emita feedback inmediato y muy próximo. En consecuencia, gracias a que dispone de información mucho más detallada sobre su proceso de aprendizaje, el alumnado se siente más apoyado y con confianza a lo largo del proceso de aprendizaje.

- Los estudiantes descubren nuevas formas de enfocar la evaluación, y sobre todo la posibilidad de implicarse en el proceso y de ser responsable de su propio aprendizaje.

- La posibilidad de conocer a los estudiantes a través de la evaluación permite al docente innovar con mayor sentido y pertinencia su acción docente.

Pero los informes también permiten constatar los siguientes inconvenientes y sus posibles vías de solución:

- Desconfianza y resistencias iniciales del alumnado hacia el sistema de evaluación: no obstante, si el sistema de evaluación se vincula a las tareas profesionales que deberá afrontar en un futuro próximo, si se explica, se argumenta y se debate con la intención de que sea más justo y transparente, es probable que éstas resistencias vayan desapareciendo progresivamente.

- El alumnado considera que estos sistemas de evaluación implican una excesiva carga de trabajo: ante este inconveniente es fundamental ha- 
cer comprender al alumnado que esta modalidad de evaluación permite identificar sus fortalezas y debilidades con respecto a su propio proceso de aprendizaje, y que, gracias a ello, será mucho más fácil concretar las acciones que le permitirán conseguir aprendizajes más profundos y mejores calificaciones. Todo ello, evidentemente, implica un esfuerzo.

- El profesorado considera que esta modalidad de evaluación conlleva, en comparación con los sistemas de evaluación tradicionales, mayor carga de trabajo y tiempo de dedicación: para afrontar este inconveniente, es fundamental disponer de espacios de reflexión entre el profesorado para analizar las ventajas e inconvenientes de un sistema u otro, y sobre todo que nadie vea un sistema de evaluación como una imposición. Tiene que darse la posibilidad de que coexistan diferentes sistemas de evaluación. La información que se obtiene de los estudiantes a través de la evaluación formativa y su utilidad para emitir valoraciones y calificaciones más contrastadas y ajustadas al nivel de aprendizaje del alumnado, hace que estos sistemas difícilmente tengan camino de regreso.

- Dificultades en la planificación y en la organización del sistema de evaluación: para minimizar esta situación es necesario que los sistemas de evaluación sean compartidos por un equipo docente, que se recojan de manera sistemática los resultados del proceso, y que año tras año se vayan haciendo pequeñas mejoras, antes que rediseñar todo el sistema por completo.

- Falta de experiencia previa por parte del profesorado y del alumnado: por este motivo es fundamental introducir pequeñas experiencias de evaluación formativa desde el inicio de la carrera y por parte del profesorado disponer de equipos docentes en los que los profesores menos experimentados en este tipo de sistemas se puedan ir familiarizando y comprobando los resultados.

- Necesidad de acreditar la veracidad de las evaluaciones de los estudiantes: para ello se requiere concretar con mayor rigor las evidencias y los indicadores asociados a los criterios de evaluación de las diferentes actividades de evaluación.

- Dificultades en el paso de la evaluación a la calificación: por este motivo se hace evidente la necesidad de negociar con el alumnado los criterios de evaluación para que éstos sirvan de orientación al alumnado a la hora de realizar las actividades. Este hecho se puede complementar con una negociación de los porcentajes en la calificación ya que esto contribuye a que los estudiantes vivan el proceso de manera transparente.

\section{INCIDENCIA DE LA INNOVACIÓN EN EL DESARROLLO PROFESIO- NAL DEL PROFESORADO UNIVERSITARIO}

Hemos mencionado con anterioridad los efectos que las innovaciones generan en las personas, puesto que, tal y como opinan Margalef y Álvarez (2005), la 
innovación en educación no se impone ni se decreta. Para estos autores la innovación tiene un fuerte componente individual. Para que se produzca el profesorado debe estar convencido de aquello que va a cambiar y de por qué va a hacerlo. Pero no basta exclusivamente con la comprensión intelectual del proceso de cambio, ni con la adquisición de nuevas habilidades, destrezas y conocimientos. También es necesario un componente afectivo y emocional. El profesorado debe de estar motivado para involucrar, entusiasmar y contagiar a los estudiantes en el proceso de cambio, y dichos procesos también necesitan para su implementación, la creación de vínculos entre profesores que permitan generar redes de entendimiento y de intercambio para que las innovaciones se hagan fuertes y lleguen a todos los miembros de la comunidad en la que se aplican.

Sin lugar a dudas, el ámbito donde mayor efecto tiene las dinámicas colaborativas derivadas de las innovaciones se sitúa en el marco del desarrollo profesional del docente universitario. El desarrollo profesional se refiere a la capacidad del profesorado a pensar por sí mismo, de analizar sus propias prácticas críticamente, desarrollando la comprensión que tiene de las mismas y de ampliar la capacidad de formular cuestiones. Pero este desarrollo no depende única y exclusivamente de un aprendizaje individual, sino que como miembro de un grupo profesional de una comunidad, también necesita de otros colegas de ese contexto específico (Gil, 2002). Asimismo, Fraile (2003), añade que la mejora no se debe centrar únicamente en conseguir una actuación docente más eficaz. También debe pretender potenciar el intercambio de conocimientos a partir de actividades colaborativas en las que se compartan experiencias dentro de un proceso de aprender juntos en un proceso continuado, y con una repercusión en la actividad laboral.

En nuestro caso, los miembros del Grupo Consolidado de Innovación en Evaluación Formativa y Participativa en la docencia universitaria de la Universidad de Barcelona, a través de los diferentes niveles de compromiso adquiridos, hemos constatado, que formar parte de un grupo de innovación contribuye de manera muy eficaz a la formación del docente universitario, y, por tanto, tiene una importante repercusión en la construcción del propio desarrollo profesional. Para recoger tales vivencias, al finalizar el curso 2006-2007 se pasó un cuestionario al profesorado participante en los grupos de innovación (Buscà et al., 2007; Julián et al., 2007). El análisis de la información obtenida, arrojó los siguientes resultados:

En primer lugar, la innovación se ha convertido en una oportunidad para revisar las propias convicciones personales sobre el proceso de enseñanza-aprendizaje seguido en la universidad. La tipificación de estas convicciones puede resultar complicada por la diversidad de puntos de partida de los diferentes miembros que componen los grupos de trabajo, y tal vez sería una osadía por parte de los autores, intentar extrapolarlas a todos ellos. Por este motivo en este articulo tan sólo se van a exponer aquellas que han resultado más significativas, y se erigen como un autentico desarrollo profesional. A saber: 
a. La reflexión sobre propia la práctica docente: reconocimiento de los puntos fuertes y débiles, y revisión de los propios discursos educativos.

b. Comprometerse con la calidad de una docencia centrada en el papel activo que tiene el alumnado en el proceso formativo y de evaluación.

c. Crecimiento personal y profesional (teórico y práctico).

d. Actualización docente, y la posibilidad de dar nombre y fundamentar las acciones docentes que ya se venían haciendo de forma intuitiva e individual.

e. Compartir conocimiento, materiales y lecturas de alto interés para la formación docente.

f. Valorar y aplicar el trabajo de colaboración entre iguales.

g. "Dar el salto" para utilizar las TIC en el proceso de enseñanza-aprendizaje.

En segundo lugar, las tareas desarrolladas en el grupo, han permitido movilizar estas convicciones, y han posibilitado asumir ciertas estrategias dentro de nuestras aulas universitarias, y en concreto en relación a la evaluación:

a. Unificar criterios en torno a la conceptualización de la evaluación formativa y sus principios: adecuación, relevancia, credibilidad, integración, continuidad, viabilidad, eticidad, emancipación.

b. Analizar y valorar las actividades de evaluación llevadas a cabo de manera individual, e incorporar la evaluación formativa al proceso de enseñanza-aprendizaje y algunos de los procedimientos que conlleva: autoevaluación, coevaluación, evaluación compartida, evaluación del docente, calificación dialogada, etc.

c. Diversificar el procedimiento de evaluación utilizando varias fuentes de información durante el proceso de aprendizaje.

d. Unificar criterios de evaluación en las asignaturas que abordan contenidos similares, fomentando el equipo docente dentro de las titulaciones.

e. Fomentar la participación del alumnado en la concreción de los criterios de evaluación de las asignaturas.

f. Recopilar información durante el proceso para hacer de la evaluación un proceso sistemático y riguroso susceptible de compartirse con otros docentes, y alumnado.

g. Adaptarse a los cambios que requiere el EEES y ajustar la dedicación del alumnado de la asignatura a los créditos ECTS que tiene asignados.

h. Desarrollar la guía docente de la asignatura con una serie de requerimientos: instrumentos de seguimiento del proceso de aprendizaje; sistematización del proceso evaluador; referencia al modelo metodológico seguido.

i. Fomentar los espacios comunes de docencia para abordar conceptos desde asignaturas diferentes. Aplicación de criterios transversales e interdisciplinares en el proceso formador. 
j. Introducir nuevos instrumentos para el desarrollo del proceso de enseñanza-aprendizaje. Estos instrumentos están muy vinculados al proceso de evaluación, y a lo que implica la recogida de información durante el desarrollo de las asignaturas. Se destacan los siguientes: tutorías ECTS, portafolio, exámenes, fichas de prácticas, informes de prácticas, rúbricas y fichas de seguimiento del trabajo del alumnado, entrevistas, diarios, etc.

Finalmente, también conviene destacar la importancia del trabajo en colaboración. Este apoyo se establece a tres niveles: a nivel de local (mediante las reuniones de trabajo de los diferentes subgrupos que están desarrollando proyectos en el mismo grupo de innovación); a nivel nacional (reuniones anuales de todos los miembros que forman parte de la Red Nacional de Evaluación Formativa y Compartida) y a nivel de relación entre redes locales (mediante reuniones entre miembros de dos o tres redes locales diferentes para llevar a cabo proyectos específicos). El trabajo en colaboración ha permitido:

a. Relacionarse con otros profesionales de la docencia universitaria, superar el aislamiento, compartir y contrastar experiencias, intercambiar información, resolver dudas y superar dificultades.

b. Estimular y motivar la práctica diaria.

c. Participar en proyectos compartidos elaborar documentos para difundir la experiencia (comunicaciones, capítulos de libro...)

d. Conformar equipos docentes comprometidos con objetivos similares y de gran afinidad.

e. Orientación al profesorado novel.

En definitiva, la experiencia de trabajo en colaboración, a través de grupos de innovación vinculados a redes nacionales, representa un proceso de formación continua altamente estimulante para todos los profesores y profesoras que han compartido este espacio de reflexión y trabajo. A la luz de la información obtenida de los miembros del grupo de innovación consideran que la Red de Evaluación Formativa y Compartida representa una verdadera posibilidad de formación y desarrollo profesional ya que cuenta con las principales características que se requiere para ello (Imbernón, 1998). A saber:

1. El profesorado interviene como investigador de su práctica profesional, de manera sistemática y con sentido crítico. Las reuniones de trabajo articuladas en el Grupo de Innovación, donde ser revisan las dificultades en la acción y sobre la acción a partir de las propias necesidades individuales, desarrollan y modifican el conocimiento profesional, creando teorías y comprobando su validez y aplicación práctica.

2. El profesorado investigador se configura como un profesional reflexivo y crítico. Ramos y Del Villar (2005) consideran la reflexión como un com- 
ponente del conocimiento profesional que sirve para superar la dicotomía mostrada por el docente respecto de la teoría aprendida y asimilada por él mismo y las particularidades manifestadas en la situación práctica, que supone un análisis de la situación más una propuesta de acción basada en ese análisis y que debe convertirse en un hábito antes, durante y después de la práctica diaria. En este sentido, el plan de acción que cada profesorado lleva a la práctica como compromiso con el grupo de innovación, sirve para mejorar los problemas que acontecen en su práctica diaria, a la vez que para huir de una práctica reproductora y acrítica.

3. El centro educativo como núcleo del desarrollo profesional. La formación en el centro no significa únicamente una situación física, sino la posibilidad de realizar una formación desde dentro (Imbernón, 1998), de forma consensuada y contextualizada. En este sentido, el trabajo con el grupo de innovación implica repensar las propias necesidades y la puesta en marcha de innovaciones de carácter colaborativo, a partir de las situaciones problemáticas vividas.

4. Una formación autónoma y colaborativa con otros docentes con experiencias y problemas similares. Los grupos de Innovación vinculados con la red, y que trabajan en planes de acción consensuados posibilitan la implantación de una cultura profesional basada en la colaboración, necesaria para un cambio en la cultura docente. Trabajar de forma coordinada, según González Álvarez y Asensio (2006), fomenta los procesos de reflexión sobre la actuación docente y permite el intercambio entre profesionales que se enfrentan a una misma labor. Además, consigue incrementar la motivación generando fuertes sentimientos dentro del equipo de trabajo, el cual se completa y se enriquece con las aportaciones de los compañeros. En definitiva, se posibilita una interacción con el resto del profesorado implicado, en donde las decisiones se comparten y las responsabilidades se distribuyen.

Finalmente, no podemos olvidar el papel que los ciclos de investigaciónacción han jugado como elemento de cohesión del funcionamiento de los Grupos de innovación vinculados a la Red. Se han erigido como una forma de indagación del profesorado para mejorar sus acciones docentes y profesionales, revisando su práctica a la luz de evidencias obtenidas de los datos y del juicio crítico de otras personas (Latorre, 2003). La investigación-acción es un importante procedimiento para la formación y el desarrollo profesional del profesorado. Gracias a la acción cooperativa que implica y al trabajo en equipo que fomenta, permite que el profesorado oriente, corrija y evalúe sus problemas y, por extensión, la toma decisiones para mejorar, analizar o cuestionar la práctica social o educativa. El profesorado se forma y desarrolla cuando adquiere un mayor conocimiento de la compleja situación en la que su enseñanza se desenvuelve. Para esto, debe unir teoría y práctica, experiencia y reflexión, acción y pensamiento, tanto para su desarrollo personal como profesional (Imbernón, 1998). 
Desde el momento en que se plantea una nueva práctica de enseñanza y evaluamos sus resultados, en cierta manera, puede decirse que se ha entrado a formar parte de la investigación educativa (Benito, 2005). El trabajo de los grupos de innovación que conforman o están vinculados a la Red de Evaluación Formativa y Compartida a través de la reflexión, el intercambio de experiencias, la puesta en marcha de un plan de acción y su posterior análisis de resultados, contribuye al conocimiento profesional de los docentes en tres campos en concreto: el personal, el teórico y el práctico, que se convierte en una forma muy enriquecedora de desarrollo profesional. (Buscà et al., 2007; Julián et al., 2007).

\section{REFERENCIAS}

Benito, A. (2005). Investigar en docencia. En A. Benito \& A. Cruz (Eds.), Nuevas claves para la Docencia Universitaria en el Espacio Europeo de Educación Superior (pp. 125-135). Madrid: Narcea.

Buscà, F., Martínez Mínguez, L., Martín, M., Camerino, O., Peire, T., Julián, J. A., Capllonch, M., Padrós, M., y Estrada, J. (2007). La red de evaluación formativa y compartida en docencia universitaria como formación permanente $y$ desarrollo profesional del docente universitario. Comunicación presentada en las II Jornadas de Evaluación Formativa en la Docencia Universitaria, La Laguna, España.

De la Torre, S. (1997). Innovación educativa. Madrid: Dikinson.

Fraile, A. (2003). Modelos y tradiciones en la formación del profesorado de Educación Física. En A. Fraile Aranda (Ed.), Didáctica de la Educación Física (pp. 291-314). Madrid: Biblioteca nueva.

Gil, P. (2002). La construcción del saber profesional. En F. Sánchez Bañuelos (Ed.), Didáctica de la Educación Física (pp. 301-326). Madrid: Prentice Hall.

González Álvarez, A., y Asensio, A. (2006). La actuación coordinada del profesorado universitario. En B. Learreta (Ed.), La coordinación del profesorado ante las demandas del Espacio Europeo de Educación Superior (pp.29-44). Madrid: Universidad Europea de Madrid.

Gros, B. (2006). La investigación sobre docencia universitaria: retos e iniciativas. En M. Martínez y S. Carrasco (Eds.), Propuestas para el cambio docente en la universidad (pp. 33-47). Barcelona: Octaedro/ICE UB.

Gros, B., y Lara, P. (2009). Estrategias de innovación en la educación superior: El caso de la Universitat Oberta de Catalunya. Revista Iberoamericana de Educación, 29, 223-245.

Hannan, A., y Silver, H. (2005). La innovación en la enseñanza superior. Enseñanza, aprendizajes y culturas institucionales. Madrid: Narcea.

Imbernón, F. (1998). La formación y el desarrollo profesional del profesorado: hacia una nueva cultura profesional (3a ed.). Barcelona: Graó.

Julián, J. A., Capllonch, M., Martínez Mínguez, L., Padrós, M., Buscà, F., y Peire, T. (2007). La red de evaluación formativa y compartida en docencia universitaria como innovación y ámbito de formación permanente del profe- 
sorado. Revisión de la experiencia y resultados encontrados. Comunicación presentada en el I Congreso Internacional Nuevas Tendencias en la Formación Permanente del Profesorado, Barcelona, España.

Latorre, A. (2003). La investigación-acción. Conocer y cambiar la práctica educativa. Barcelona: Graó.

López Pastor, V.M., Martínez-Muñoz, L. F., y Julian, J.A. (2007). La red de Evaluación Formativa, Docencia Universitaria y Espacio Europeo de Educación Superior (EEES). Presentación del Proyecto, grado de desarrollo y primeros resultados. Red-U. Revista de Docencia Universitaria, 2. Obtenido de http:// www.redu.um.es/red_U/2

Margalef, L., y Álvarez Méndez, J. M. (2005). La formación del profesorado universitario para la innovación en el marco de la integración del Espacio Europeo de Educación Superior. Revista de Educación, 337, 51-70.

Ramos, L. A., y Del Villar, F. (2005). Los procesos de reflexión del profesorado. En L. A. Ramos y F. Del Villar (Eds.), La enseñanza de la educación física. Análisis de las primeras experiencias docentes. (pp. 61-86). Madrid: Síntesis.

Vallés, C., Ureña, N. y Ruiz, E. (2011). La Evaluación Formativa en Docencia Universitaria. Resultados Globales de 41 estudios de caso. REDU. Revista de Docencia Universitaria, 9(1), 135-158. Obtenido de http://redaberta.usc.es/ redu/index.php/REDU

Zaragoza, J., Luis, J.C. y Manrique, J.C. (2008). Experiencias de innovación en docencia universitaria: resultados de la aplicación de sistemas de evaluación formativa. REDU. Revista de Docencia Universitaria, 4, 1-33. Obtenido de http://www.redu.um.es/red_U4 
